\title{
A Tunable Resolution MUSIC Algorithm for Interharmonics Analysis
}

\author{
Ming Zhang1 ${ }^{*}$, Xiang Zhang1, Heng Yao ${ }^{1}$, Shunfan $\mathrm{He}^{2}$ \\ ${ }^{1}$ School of Electronic and Electrical Engineering, Wuhan Textile University, Wuhan, China \\ ${ }^{2}$ College of Computer Science, South Central University for Nationalities, Wuhan, China \\ Email: ${ }^{*}$ zhangming@wtu.edu.cn
}

How to cite this paper: Zhang, M., Zhang, X., Yao, H. and He, S.F. (2017) A Tunable Resolution MUSIC Algorithm for Interharmonics Analysis. Journal of Power and Energy Engineering, 5, 1-13.

https://doi.org/10.4236/jpee.2017.59001

Received: July 27, 2017

Accepted: August 28, 2017

Published: August 31, 2017

Copyright $\odot 2017$ by authors and Scientific Research Publishing Inc. This work is licensed under the Creative Commons Attribution International License (CC BY 4.0).

http://creativecommons.org/licenses/by/4.0/

\begin{abstract}
The harmonic and interharmonic analysis recommendations are contained in the latest IEC standards on power quality. Measurement and analysis experiences have shown that great difficulties arise in the interharmonic detection and measurement with acceptable levels of accuracy. In order to improve the resolution of spectrum analysis, the traditional method (e.g. discrete Fourier transform) is to take more sampling cycles, e.g. 10 sampling cycles corresponding to the spectrum interval of $5 \mathrm{~Hz}$ while the fundamental frequency is $50 \mathrm{~Hz}$. However, this method is not suitable to the interharmonic measurement, because the frequencies of interharmonic components are non-integer multiples of the fundamental frequency, which makes the measurement additionally difficult. In this paper, the tunable resolution multiple signal classification (TRMUSIC) algorithm is presented, which the spectrum can be tuned to exhibit high resolution in targeted regions. Some simulation examples show that the resolution for two adjacent frequency components is usually sufficient to measure interharmonics in power systems with acceptable computation time. The proposed method is also suited to analyze interharmonics when there exists an undesirable asynchronous deviation and additive white noise.
\end{abstract}

\section{Keywords}

Interharmonics Analysis, Tunable Resolution Multiple Signal Classification (TRMUSIC) Algorithm, Subspace Decomposition, Spectral Analysis

\section{Introduction}

Interharmonics can be thought of as the inter-modulation of the fundamental and harmonic components of the power system with any other frequency com- 
ponents and can be observed in an increasing number of loads. These loads include static frequency converters, cycloconverters, sub-synchronous converter cascades, induction motors, arc furnaces and so on [1].

A method, which is aimed to standardize the harmonic and interharmonic measurement, has been proposed by the IEC [2]. This method utilizes discrete Fourier transform (DFT) performed over a rectangular time window of exactly 10 cycles for $50 \mathrm{~Hz}$ power systems. The window width fixes the frequency resolution at $5 \mathrm{~Hz}$, so the interharmonic components that are between the bins spaced of $5 \mathrm{~Hz}$ would spill over primarily into adjacent interharmonic bins with a minimum of spill into harmonic bins. Therefore, the harmonic and interharmonic groups are introduced. The interharmonic group is defined as the RMS (Rootmean-square) value of all the interharmonic components between adjacent harmonic groups (see Figure 1).

However, the accurate estimation method of the interharmonic components has not been established yet. Many researchers have been studying new methods. For analyzing a range of the interharmonic components, researchers often use DFT and its improved algorithms to calculate amplitudes, frequencies and phases of the interharmonic components [3] [4] [5] [6] [7]. The major pitfalls in the common DFT applications are the spectral leakage and picket fence effects.

The multiple signal classification (MUSIC) algorithm exploits the noise subspace to estimate the unknown parameters of the random process, which was proposed by R. O., Schmidt [8]. This algorithm can also estimate the frequencies of complex sinusoids corrupted with additive white noise. T. Lobos et al. [9] [10] have already proposed the frequencies determination method of the harmonic components using the MUSIC algorithm. But it is difficult to estimate the frequencies of the interharmonic components.

In this paper, the tunable resolution MUSIC (TRMUSIC) algorithm is presented to estimate the parameters of interharmonics, which the spectrum can be tuned to exhibit high resolution in targeted regions. The organization of this paper is as follows. The interharmonic measurement method based on the TRMUSIC algorithm is proposed in Section 2. Then, simulation results to demonstrate the

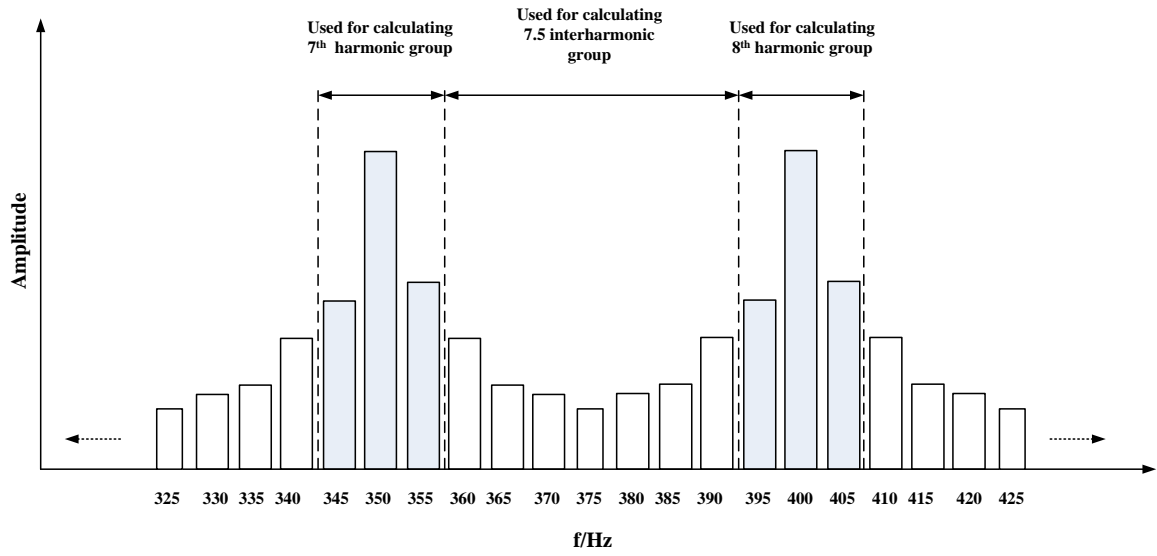

Figure 1. Harmonic and interharmonic (sub) groups. 
validity, precision feasibility and robustness of the algorithm are presented in Section 3. At last, the conclusions are given in Section 4.

\section{Trmusic Algorithm}

\subsection{Music Algorithm}

The MUSIC algorithm is an eigenvalue subspace decomposition method for estimation of the frequencies of complex sinusoids observed in additive white noise. Consider a noisy signal vector $y$ comprised of $P$ complex sinusoids modeled as

$$
y(n)=\sum_{i=1}^{P} A_{i} \mathrm{e}^{j 2 \pi f_{i} \Delta t}+e(n), n=0,1, \cdots,(N-1)
$$

with

$$
A_{i}=\left|A_{i}\right| \mathrm{e}^{j \varphi_{i}}
$$

where $\left|A_{i}\right|, \quad f_{i}$ and $\varphi_{i}$ represent the amplitude, frequency and phase of $i$-th complex sinusoid, respectively. $N$ is the number of samples in one data rectangular window, $\Delta t$ is the fixed time interval, and $e(n)$ is a zero mean Gaussian white noise vector with variance $\sigma_{n}^{2}$.

Suppose that $\boldsymbol{Y}(n)$ is the sampled set. Since it is known that $\boldsymbol{Y}(n)=[y(n), y(n+1), \cdots, y(n+N-1)]^{\mathrm{T}}$, the $\boldsymbol{Y}(n)$ can be expressed as

$$
\boldsymbol{Y}(n)=\boldsymbol{A}(f) \boldsymbol{X}(n)+\boldsymbol{E}(n)
$$

with

$$
\begin{aligned}
\boldsymbol{A}(f) & =\left[\boldsymbol{a}\left(f_{1}\right), \boldsymbol{a}\left(f_{2}\right), \cdots, \boldsymbol{a}\left(f_{P}\right)\right] \\
\boldsymbol{a}\left(f_{i}\right) & =\left[1, \mathrm{e}^{j 2 \pi f_{i} \Delta t}, \cdots, \mathrm{e}^{j 2 \pi(N-1) f_{i} \Delta t}\right]^{\mathrm{T}} \\
\boldsymbol{X}(n) & =\left[x_{1}(n), x_{2}(n), \cdots, x_{P}(n)\right]^{\mathrm{T}}
\end{aligned}
$$

where $x_{i}(n)=A_{i} \mathrm{e}^{j 2 \pi f_{i} n}$.

The auto-correlation matrix of the noisy signal $\mathbf{Y}(n)$ can be written as

$$
\boldsymbol{R}_{Y Y}=\mathrm{E}\left[\boldsymbol{Y}(n) \boldsymbol{Y}^{\mathrm{H}}(n)\right]=\boldsymbol{R}_{X X}+\boldsymbol{R}_{E E}=\boldsymbol{A} \boldsymbol{P} \boldsymbol{A}^{\mathrm{H}}+\sigma_{n}^{2} \boldsymbol{I}
$$

where $\mathrm{E}$ denotes the expectation, $\mathrm{H}$ denotes the Domitian transpose and $\boldsymbol{P}=\mathrm{E}\left[\boldsymbol{X}(n) \boldsymbol{X}^{\mathrm{H}}(n)\right]$ is the diagonal matrix. In addition, $\boldsymbol{R}_{X X}=\boldsymbol{A} \boldsymbol{P} \boldsymbol{A}^{\mathrm{H}}$ and $\boldsymbol{R}_{E E}=\sigma_{n}^{2} \boldsymbol{I}$ are the auto-correlation matrices of the signal and noise processes respectively, as follows

$$
\begin{gathered}
\boldsymbol{R}_{X X}=\sum_{i=1}^{N} \lambda_{i} v_{i} v_{i}^{\mathrm{H}} \\
\boldsymbol{R}_{E E}=\sigma_{n}^{2} \sum_{i=1}^{N} v_{i} v_{i}^{\mathrm{H}}
\end{gathered}
$$

where $\lambda_{i}$ and $v_{i}$ are the eigenvalues and convector of the matrix $\boldsymbol{R}_{X X}$, respectively. So, the auto-correlation matrix of the noisy signal may be expressed as 


$$
\boldsymbol{R}_{Y Y}=\sum_{i=1}^{N} \lambda_{i} v_{i} v_{i}^{\mathrm{H}}+\sigma_{n}^{2} \sum_{i=1}^{N} v_{i} v_{i}^{\mathrm{H}}=\sum_{i=1}^{N} \mu_{i} v_{i} v_{i}^{\mathrm{H}}
$$

where $\left\{\mu_{i}=\lambda_{i}+\sigma_{n}^{2}, i=1,2, \cdots, N\right\}$ are the eigenvalues of the matrix $\boldsymbol{R}_{Y Y}$. All the eigenvalues are the real numbers and satisfy

$$
\mu_{1} \geq \mu_{2} \geq \cdots \geq \mu_{p}>\mu_{p+1}=\cdots=\mu_{N}=\sigma_{n}^{2}
$$

Furthermore, the singular value decomposition (SVD) of the matrix $\boldsymbol{R}_{Y Y}$ can be written as

$$
\boldsymbol{R}_{Y Y}=\boldsymbol{U} \Sigma \boldsymbol{V}^{\mathrm{H}}
$$

where the columns of $\boldsymbol{U}$ and $\boldsymbol{V}$ are the left and right singular vectors, respectively. $\Sigma$ is a diagonal matrix whose diagonal entries are the positive eigenvalues of $\boldsymbol{R}_{Y Y}$, and $\Sigma=\operatorname{diag}\left[\mu_{1} \mu_{2} \cdots \mu_{N}\right]$.

Then, the MUSIC spectrum is defined as [11]

$$
P_{\text {MUSIC }}(f)=\frac{1}{\sum_{i=p+1}^{N}\left|\boldsymbol{a}^{\mathrm{H}}(f) v_{i}\right|^{2}}=\frac{1}{\boldsymbol{a}^{\mathrm{H}}(f) \boldsymbol{S} \boldsymbol{S}^{\mathrm{H}} \boldsymbol{a}(f)}
$$

with

$$
\begin{gathered}
\boldsymbol{a}\left(f_{r}\right)=\left[1, \mathrm{e}^{j 2 \pi f_{r} \Delta t}, \cdots, \mathrm{e}^{j 2 \pi(N-1) f_{r} \Delta t}\right]^{\mathrm{T}} \\
\boldsymbol{S}=\left[v_{P+1} \cdots v_{N}\right]
\end{gathered}
$$

where $\boldsymbol{a}\left(f_{r}\right)$ is the complex sinusoidal vector, $f_{r}$ is the frequency resolution of the MUSIC spectral estimation, and $S$ is the matrix of convector of the noise subspace.

\subsection{The Proposed Tunable Resolution Method}

The frequency resolution $f_{r}$ of the DFT spectral estimation is low when the sampling time $t_{c}$ (it is also the width of rectangular window) is short because $f_{r}=1 / t_{c}=f_{s} / N$, where $f_{s}$ is the sampling frequency. The frequency resolution can be improved by increasing the number of frequency points, but it may increase the calculation time. The MUSIC algorithm is known as a high-resolution frequency estimation method, however, its frequency resolution $f_{r}$ is invariable, which doesn't allow the best frequency resolution in a dynamic signal.

Here, a method of obtaining spectral interpolation data on the use of tunable factor $\Delta$ is presented. According to the required frequency resolution of interharmonics analysis, the tunable factor $\Delta$ is decided. Furthermore, the frequency resolution can be adapt adjusted by changing the tunable factor $\Delta$. Thus, $\boldsymbol{a}\left(f_{r}\right)$ in Equation (14) can be expressed as

$$
\boldsymbol{a}\left(f_{r}^{\prime}\right)=\left[1, \mathrm{e}^{j 2 \pi f_{r}^{\prime} \Delta t}, \cdots, \mathrm{e}^{j 2 \pi(N-1) f_{r}^{\prime} \Delta t}\right]^{\mathrm{T}}
$$

with

$$
f_{r}^{\prime}=[0,1, \cdots, k] \cdot \frac{f_{r}}{\Delta}, k=\Delta
$$


where $f_{r}^{\prime}$ is the frequency bin sets with the tunable factor $\Delta, f_{r} / \Delta$ is the updated frequency resolution, and $\Delta$ must be an integer, as shown in Figure 2. Therefore, such data will replace the initial data for the frequencies estimation.

\subsection{Denouncing Algorithm Based on Cross-Spectral Estimation}

The most important step is to estimate the signal subspace dimension $P$ for spectral analysis. However, the noise yields an inconsistent estimation that tends to estimate the number of peaks in the range profile. To overcome this problem a denouncing algorithm based on cross-spectral estimation has proposed.

Assume two signal sequences

$$
\begin{gathered}
\boldsymbol{Y}(n)=[y(n), y(n+1), \cdots, y(n+N-1)]^{\mathrm{T}} \\
\boldsymbol{Y}^{\prime}(n)=\boldsymbol{Y}(n+m)=[y(n+m), y(n+m+1), \cdots, y(n+N+m-1)]^{\mathrm{T}}
\end{gathered}
$$

then

$$
\begin{gathered}
\boldsymbol{Y}(n)=\boldsymbol{A}(f) \boldsymbol{X}(n)+\boldsymbol{E}(n) \\
\boldsymbol{Y}^{\prime}(n)=\boldsymbol{A}(f) \boldsymbol{X}^{\prime}(n)+\boldsymbol{E}^{\prime}(n)=\boldsymbol{A}(f) \Omega \boldsymbol{X}(n)+\boldsymbol{E}(n+m)
\end{gathered}
$$

with $\Omega=\operatorname{diag}\left(\mathrm{e}^{j 2 \pi f_{1} m \Delta t}, \mathrm{e}^{j 2 \pi f_{2} m \Delta t}, \cdots, \mathrm{e}^{j 2 \pi f_{p} m \Delta t}\right)$. Thus, the cross-correlation matrix $\boldsymbol{R}_{Y Y^{\prime}}$ of the noisy signal $\boldsymbol{Y}(n)$ and $\boldsymbol{Y}^{\prime}(n)$ is

$$
\boldsymbol{R}_{Y Y^{\prime}}=E\left[\boldsymbol{Y}(n) \boldsymbol{Y}^{\prime \mathrm{H}}(n)\right]=\boldsymbol{R}_{X X^{\prime}}+\boldsymbol{R}_{E E^{\prime}}+\boldsymbol{R}_{X E^{\prime}}+\boldsymbol{R}_{E X^{\prime}}
$$

From Equation (20), the matrix $\boldsymbol{R}_{Y Y^{\prime}}$ is composed of $\boldsymbol{R}_{X X^{\prime}}$, which is the cross-correlation matrix of the clean harmonic signal sequences, $\boldsymbol{R}_{E E^{\prime}}$, which is the cross-correlation matrix of the noise sequences, and two other cross-correlation terms $\boldsymbol{R}_{X E^{\prime}}$ and $\boldsymbol{R}_{E X^{\prime}}$. For two noise sequences assumed to be independent, we can get $\boldsymbol{R}_{E E^{\prime}}=0$ [12]. Typically it is assumed that the clean harmonic signal and noise sequences are uncorrelated. This has the effect of removing the cross-correlation terms $\boldsymbol{R}_{X E^{\prime}}$ and $\boldsymbol{R}_{E X^{\prime}}$ from the matrix $\boldsymbol{R}_{Y Y^{\prime}}$. Therefore, the matrix $\boldsymbol{R}_{Y Y^{\prime}}$ simplifies to

$$
\boldsymbol{R}_{Y Y^{\prime}}=E\left[\boldsymbol{Y}(n) \boldsymbol{Y}^{\prime \mathrm{H}}(n)\right]=\boldsymbol{R}_{X X^{\prime}}=\boldsymbol{A} \boldsymbol{P} \Omega^{\mathrm{H}} \boldsymbol{A}^{\mathrm{H}}
$$

where $\boldsymbol{X}^{\prime}(n)=\boldsymbol{X}(n) \Omega^{\mathrm{H}}$. Equation (21) showsthat the cross-correlation matrix $\boldsymbol{R}_{Y Y^{\prime}}$ of the noisy signal $\boldsymbol{Y}(n)$ and $\boldsymbol{Y}^{\prime}(n)$ is correlative to the noise. Thus, the SVD of the matrix $\boldsymbol{R}_{Y Y^{\prime}}$ can be writtenas [13].

$$
\boldsymbol{R}_{Y Y^{\prime}}=\boldsymbol{U}^{\prime} \Sigma^{\prime} \boldsymbol{V}^{\prime \mathrm{H}}=\left[\begin{array}{ll}
\boldsymbol{U}_{1}^{\prime} & \boldsymbol{U}_{2}^{\prime}
\end{array}\right]\left[\begin{array}{cc}
\Sigma^{\prime} & 0 \\
0 & 0
\end{array}\right]\left[\begin{array}{ll}
\boldsymbol{V}_{1}^{\prime} & \boldsymbol{V}_{2}^{\prime}
\end{array}\right]^{\mathrm{H}}
$$

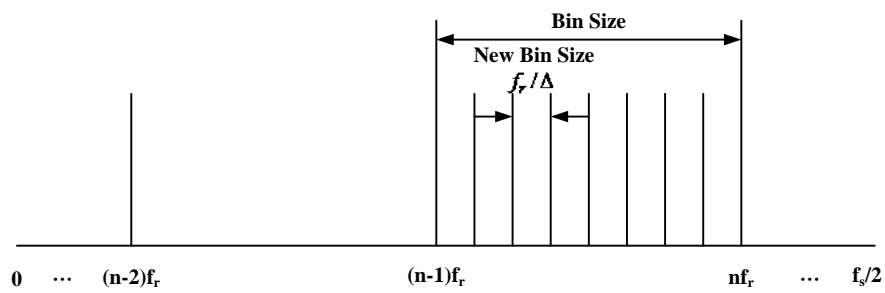

Figure 2. Resolution of MUSIC spectrum. 
where the columns of $U^{\prime}$ and $V^{\prime}$ are the left and right singular vectors respectively, and $\boldsymbol{U}^{\prime}=\left[\begin{array}{ll}\boldsymbol{U}_{1}^{\prime} & \boldsymbol{U}_{2}^{\prime}\end{array}\right], \quad \boldsymbol{V}^{\prime}=\left[\begin{array}{ll}\boldsymbol{V}_{1}^{\prime} & \boldsymbol{V}_{2}^{\prime}\end{array}\right], \quad \Sigma^{\prime}=\operatorname{diag}\left[\lambda_{1}, \lambda_{2}, \cdots, \lambda_{P}\right]$.

In a real application, the cross-correlation matrix $\boldsymbol{R}_{Y Y^{\prime}}$ is not known, and it should be estimated with sampled data as follows

$$
\hat{\boldsymbol{R}}_{Y Y^{\prime}}=\frac{1}{N} \sum_{n=0}^{N-1} \boldsymbol{Y}(n) \boldsymbol{Y}^{\prime \mathrm{H}}(n)=\frac{1}{N} \sum_{n=0}^{N-1} \boldsymbol{Y}(n) \boldsymbol{Y}^{\mathrm{H}}(n+m)
$$

The matrix $\boldsymbol{R}_{Y Y^{\prime}}$ also takes the form

$$
\hat{R}_{Y Y^{\prime}}=\left[\begin{array}{cccc}
r_{11} & r_{12} & \cdots & r_{1 N} \\
r_{21} & r_{22} & \cdots & \\
\vdots & \vdots & \ddots & \vdots \\
r_{N 1} & r_{2 N} & \cdots & r_{N N}
\end{array}\right]
$$

where each element $r_{i j}(i, j=1,2, \cdots, N)$ is a positive real number such that $r_{i j}=\frac{1}{N} \sum_{n=0}^{N-1} y(n+i) \cdot y^{\prime}(n+j)$.

So, the Equation (23) can be used to estimate the signal subspace dimension $P$ accurately. For example, because zero coefficients are concentrated in the higher-lags, a noise robust algorithm by using only the lower-lags of the matrix $\hat{\boldsymbol{R}}_{Y Y^{\prime}}$ can be designed to estimate the signal subspace dimension $P$. Therefore, Equation (13) can be rewritten as

$$
P_{\text {MUSIC }}(f)=\frac{1}{\sum_{i=P+1}^{N}\left|\boldsymbol{a}^{\mathrm{H}}(f) v_{i}^{\prime}\right|^{2}}=\frac{1}{\boldsymbol{a}^{\mathrm{H}}(f) \boldsymbol{S}^{\prime} \boldsymbol{S}^{\prime \mathrm{H}} \boldsymbol{a}(f)}
$$

where $S^{\prime}$ is the updated matrix of convector of the noise subspace.

\subsection{Estimation Method of the Amplitude and Phase of the Harmonic and Interharmonic Components}

The frequencies of the harmonic and interharmonic components can be estimated from the peak location of the MUSIC spectrum, i.e., the frequencies $\left\{f_{i}, i=1,2, \cdots, p\right\}$ can be derived from the horizontal coordinate of the peak point of $P_{\text {MUSIC }}(f)$. After the estimation of the frequencies, the signal subspace dimension $P$ of the input signal can also be estimated. In a real application, $\hat{y}(n)$ is not known. Because the amplitude of the noise is very smaller than that of the signal components, let $y(n)$ replaces $\hat{y}(n)$. Then, the estimation $\hat{y}(n)$ of $y(n)$ can be represented by

$$
\hat{y}(n)=y(n)-e(n)=\sum_{i=1}^{P} \mathrm{e}^{j 2 \pi f_{i} n \Delta t} \cdot A_{i}
$$

where $n=0,1, \cdots,(N-1)$, i.e.,

$$
\left[\begin{array}{cccc}
1 & \cdot & \cdots & 1 \\
\mathrm{e}^{j 2 \pi f_{1} \Delta t} & \mathrm{e}^{j 2 \pi f_{2} \Delta t} & \cdots & \mathrm{e}^{j 2 \pi f_{p} \Delta t} \\
\vdots & \vdots & \ddots & \vdots \\
\mathrm{e}^{j 2 \pi f_{1}(N-1) \Delta t} & \mathrm{e}^{j 2 \pi f_{1}(N-1) \Delta t} & \cdots & \mathrm{e}^{j 2 \pi f_{1}(N-1) \Delta t}
\end{array}\right]\left[\begin{array}{c}
A_{1} \\
A_{2} \\
\vdots \\
A_{P}
\end{array}\right]=\left[\begin{array}{c}
\hat{y}(0) \\
\hat{y}(1) \\
\vdots \\
\hat{y}(N-1)
\end{array}\right]
$$


Equation (27) can be used to solve least squares for the coefficients $\left\{A_{i}, i=1,2, \cdots, p\right\}$ using only the available data samples [14]. Therefore, the amplitude and phase of the components can be obtained from $\left\{A_{i}, i=1,2, \cdots, p\right\}$, as follows

$$
\begin{gathered}
\left|A_{i}\right|=\sqrt{\left(\operatorname{Re}\left(A_{i}\right)\right)^{2}+\left(\operatorname{Im}\left(A_{i}\right)\right)^{2}} \\
\varphi_{i}=\arctan \left(\frac{\operatorname{Im}\left(A_{i}\right)}{\operatorname{Re}\left(A_{i}\right)}\right)
\end{gathered}
$$

where $\operatorname{Re}(\cdot)$ returns the real part of the argument, and $\operatorname{Im}(\cdot)$ returns the imaginary part of the argument.

\section{Simulation Results}

Three cases are performed in Matlab to demonstrate the effectiveness of the proposed algorithm.

\subsection{Case 1}

In practice, the fundamental frequency often deviates from its nominal value. In the first simulation, the fundamental frequency is set to $49 \mathrm{~Hz}$, and the signal is

$$
y(t)=0.1 \sin \left(2 \pi 44 t+30^{\circ}\right)+\sin \left(2 \pi 49 t-45^{\circ}\right)+0.2\left(2 \pi 57 t+60^{\circ}\right)+e(t)
$$

the sampling frequency $f_{s}$ is $6400 \mathrm{~Hz}$, the number of samples $N$ is $1280(10$ cycles), the noise variance $\sigma_{n}^{2}$ is 0.1 , the tunable factor $\Delta$ is set to 5 . It can be seen from Equation (30) that includes the interharmonic components of $44 \mathrm{~Hz}$ and $57 \mathrm{~Hz}$. Figure 3 displays the spectrums of MUSIC algorithm based on autospectral estimation, TRMUSIC algorithm based on cross-spectral estimation, and DFT algorithm when the width of rectangular window is $0.2 \mathrm{~s}$ (10 cycles), respectively.

In the second simulation, the fundamental frequencies is set to $50.2 \mathrm{~Hz}$, and the signal is

$$
y(t)=0.1 \sin \left(2 \pi 44.5 t+30^{\circ}\right)+\sin \left(2 \pi 50.2 t-45^{\circ}\right)+0.2\left(2 \pi 57.3 t+60^{\circ}\right)+e(t)(31)
$$

the sampling frequency $f_{s}$ is $6400 \mathrm{~Hz}$, the number of samples $N$ is 1280 , the noise variance $\sigma_{n}^{2}$ is 0.1 , the tunable factor $\Delta$ is set to 50 . It can be seen from Equation (31) that includes the interharmonic components of $44.5 \mathrm{~Hz}$ and 57.3 Hz. Figure 4 displays the spectrums of the MUSIC algorithm based on autospectral estimation, TRMUSIC algorithm based on cross-spectral estimation, and DFT algorithm when the width of rectangular window is $0.2 \mathrm{~s}$, respectively.

In Figure 3 and Figure 4, the results demonstrate that the TRMUSIC algorithm is not affected by asynchronous sampling, while the MUSIC algorithm performs badly in that the peaks of the MUSIC spectrum are not sharp, and the DFT algorithm produces large spectral leakage and it even cannot detect most of the true frequencies of components in the signal. For example, the second simulation requires that the best frequency resolution is $0.1 \mathrm{~Hz}$, however, the frequency resolution of the MUSIC and DFT algorithm is $5 \mathrm{~Hz}$, respectively. When 


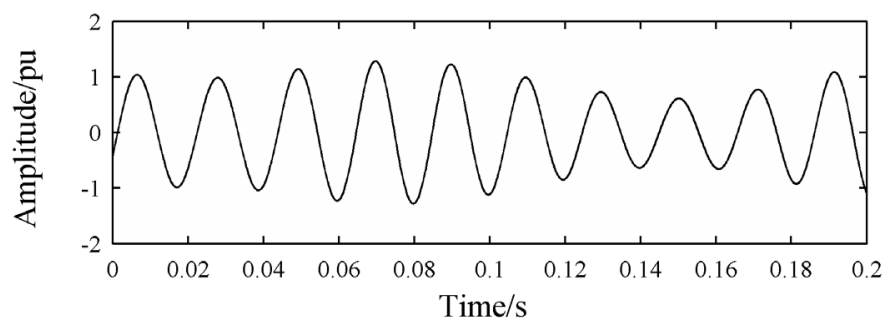

(a)

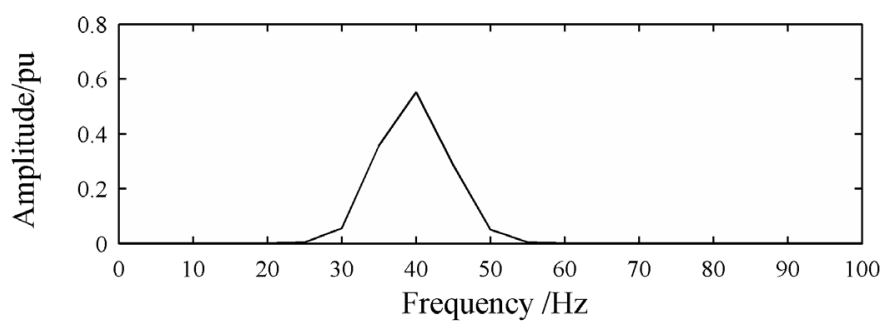

(b)

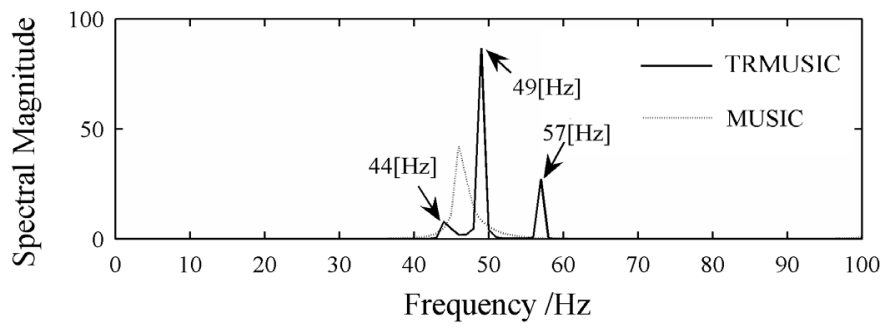

(c)

Figure 3. Spectrums of DFT, MUSIC and TRMUSIC algorithm for the first simulation: (a) Original signal; (b) DFT spectrum; (c) MUSIC and TRMUSIC spectrum.

the tunable factor $\Delta$ is set to 50, the frequency resolution of the TRMUSIC algorithm is $0.1 \mathrm{~Hz}$. It is seen from Figure 4 that the TRMUSIC spectrum has sharp peaks. Figure 4 shows the accurate frequencies estimation of the fundamental and interharmonic components $(44.5 \mathrm{~Hz}, 50.2 \mathrm{~Hz}, 57.3 \mathrm{~Hz})$. The corresponding estimation results are listed in Table 1.

\subsection{Case 2}

In this section, simulations are presented to demonstrate the anti-noise performance of TRMUSIC algorithm based on cross-spectral estimation comparing to that of the MUSIC algorithm based on auto-spectral estimation. When the signal represented by Equation (31) is contaminated with additive noise ( $\mathrm{SNR}=10 \mathrm{~dB})$, the results of four simulations are shown in Figure 5. It can be seen that the TRMUSIC algorithm based on cross-spectral estimation is only slightly affected by additive noise because the pseudo-peaks can locate steadily in the corresponding frequency bins. Although the TRMUSIC spectrums are variable in the magnitudes, the estimation results are quite accurate. Therefore, the TRMUSIC algorithm based on cross-spectral estimation has satisfying results in analyzing noisesmeared signals. 


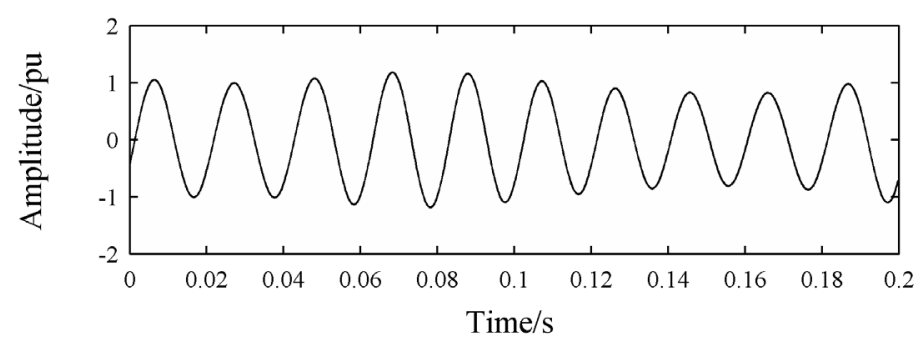

(a)

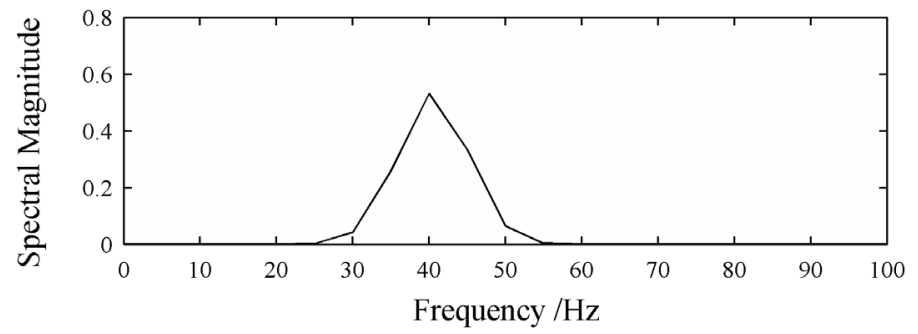

(b)

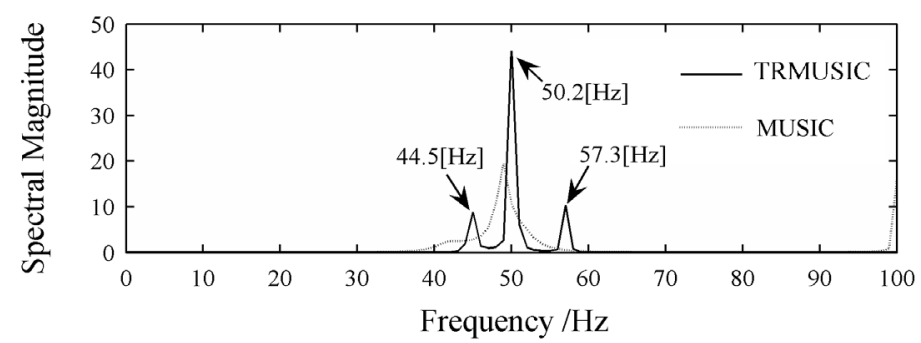

(c)

Figure 4. Spectrums of DFT, MUSIC and TRMUSIC algorithm for the second simulation: (a) Original signal; (b) DFT spectrum; (c) MUSIC and TRMUSIC spectrum.

Table 1. Results of fundamental and interharmonic components measurement.

\begin{tabular}{ccccccc}
\hline & \multicolumn{2}{c}{ Frequency $[\mathrm{Hz}]$} & \multicolumn{2}{c}{ Amplitude $[\mathrm{Pu}]$} & \multicolumn{2}{c}{ Phase [degree] } \\
\cline { 2 - 7 } Case & $\begin{array}{c}\text { True } \\
\text { Values }\end{array}$ & $\begin{array}{c}\text { TRMUSIC } \\
\text { Estimation } \\
\text { Values }\end{array}$ & $\begin{array}{c}\text { True } \\
\text { Values }\end{array}$ & $\begin{array}{c}\text { TRMUSIC } \\
\text { Estimation } \\
\text { Values }\end{array}$ & $\begin{array}{c}\text { True } \\
\text { Values }\end{array}$ & $\begin{array}{c}\text { TRMUSIC } \\
\text { Estimation } \\
\text { Values }\end{array}$ \\
\hline \multirow{2}{*}{1} & 44 & 44 & 0.1 & 0.099 & 30 & 30.7237 \\
& 49 & 49 & 1 & 1.001 & -45 & -44.8523 \\
& 57 & 57 & 0.2 & 0.199 & 60 & 60.6537 \\
& 44.5 & 44.5 & 0.1 & 0.098 & 30 & 30.6235 \\
& 50.2 & 50.2 & 1 & 1.002 & -45 & -44.7641 \\
& 57.3 & 57.3 & 0.2 & 0.1998 & 60 & 60.5574 \\
\hline
\end{tabular}

In contrast, the MUSIC algorithm based on auto-spectral estimation has large errors of the signal subspace dimension $P$ estimation; in consequence, the amplitude and phase estimation of the harmonic and interharmonic components may produce big deviation. 


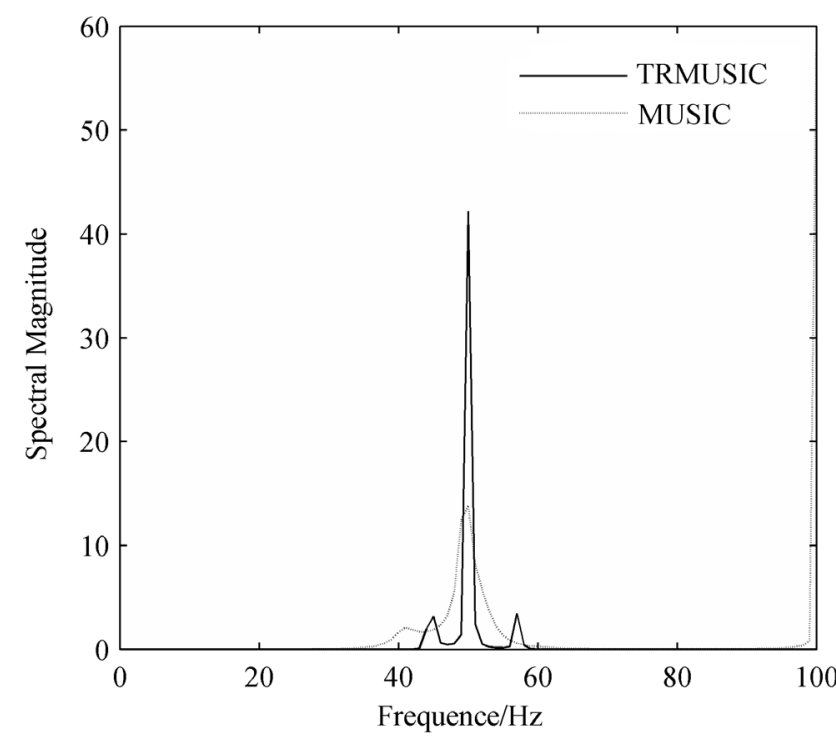

(a)

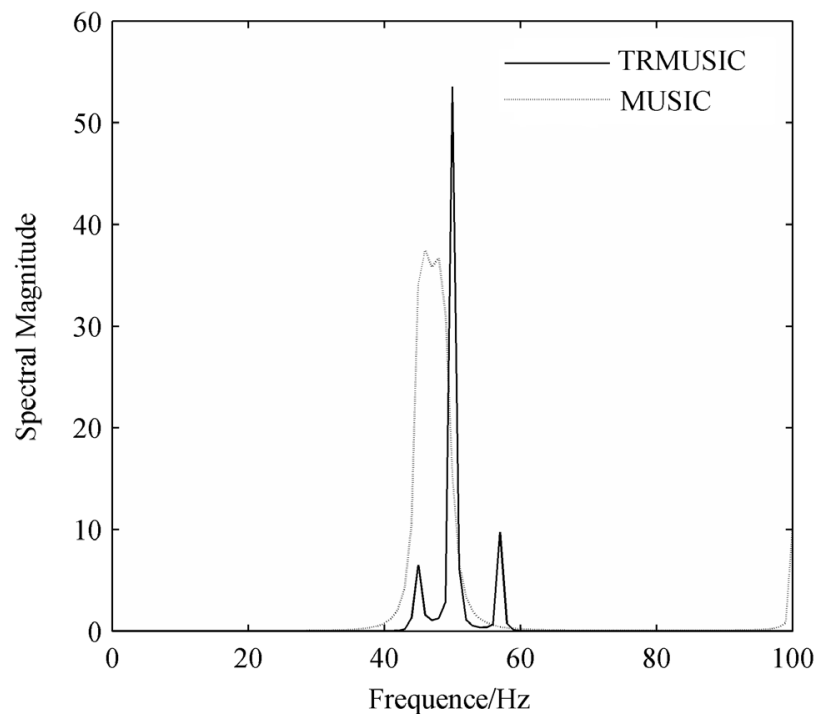

(c)

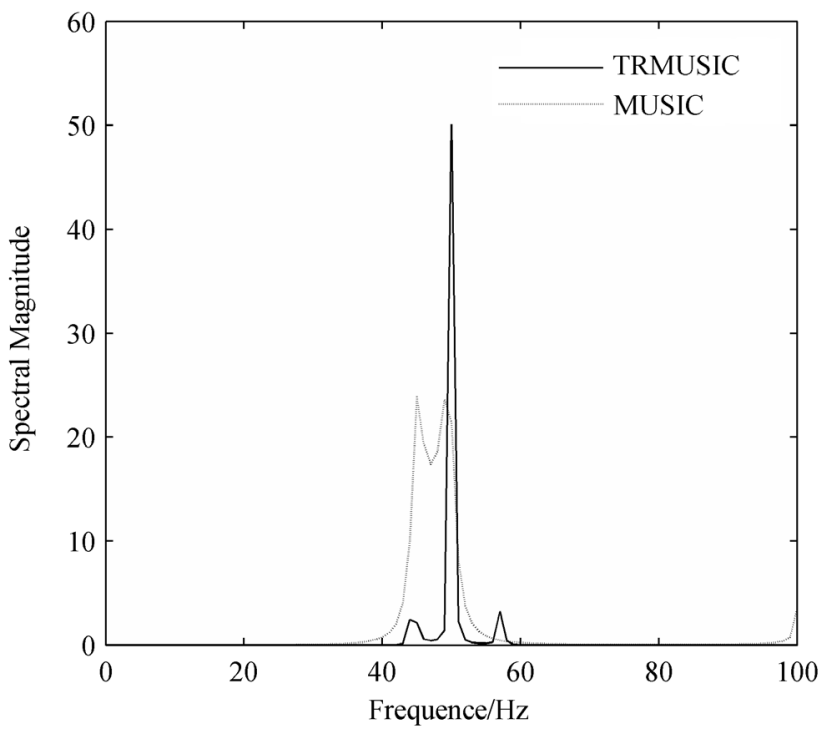

(b)

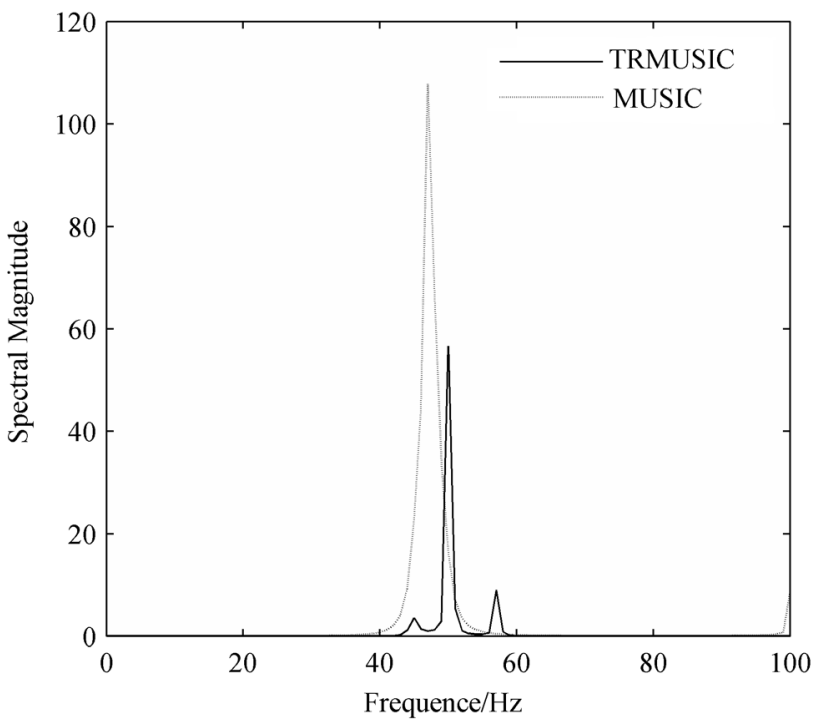

(d)

Figure 5. Comparison between the auto-spectral and cross-spectral estimation algorithm: (a) Simulation 1; (b) Simulation 2; (c) Simulation 3; (d) Simulation 4.

\subsection{Case 3}

This simulation analyzes the harmonics in the $\mathrm{AC} / \mathrm{DC} / \mathrm{AC}$ converter system.

The AC/DC/AC converter system is a typical source of interharmonics [7] [15]. The inter-harmonic frequencies of the input current derive from the modulation of the converter harmonic components of operated by the rectifier harmonics (see Figure 6). The simulation model of the AC/DC/AC converter system is established in Matlab/Simulation. The parameters of the model are as follows. The parameters of the ac supply are $U_{s}=25 / \sqrt{3} \mathrm{KV}, L_{s}=1 \mathrm{H}, R_{s}=20 \Omega$. The inductance of the dc side is $L_{d c}=5 \mathrm{~m} \mathrm{H}$. The parameters of load are $L_{l}=50 \mathrm{~m} \mathrm{H}, R_{l}=10 \Omega$.The parameters of transformer are $25 \mathrm{KV} / 600 \mathrm{~V}$, 


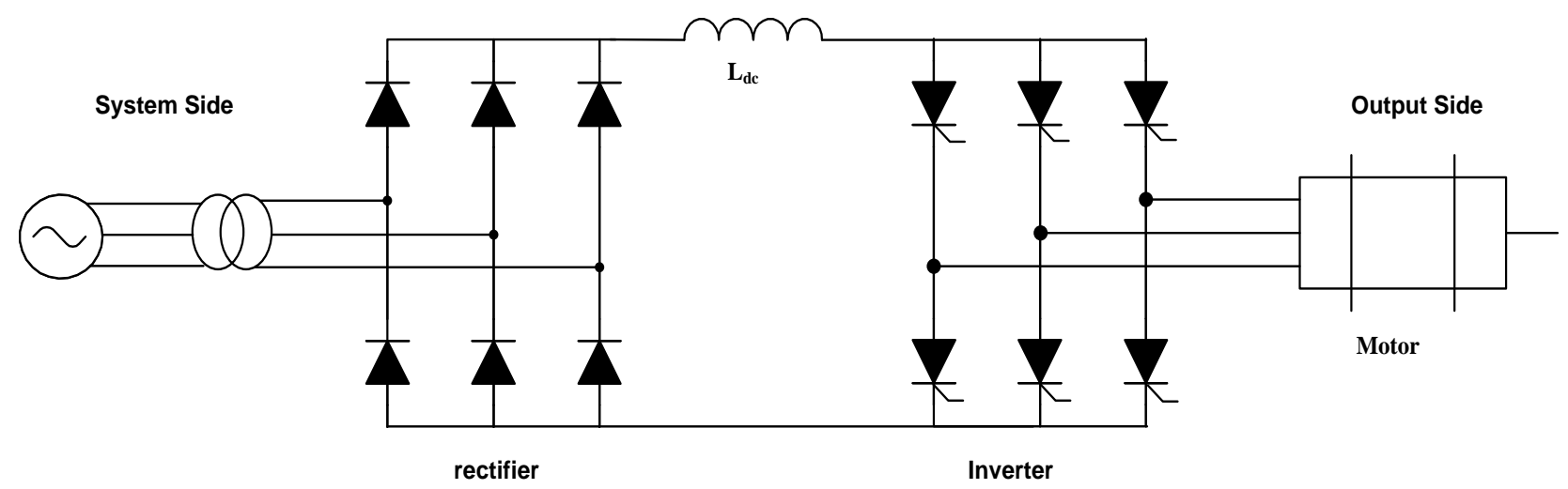

Figure 6. AC/DC/AC converter system.

Y-Y connection. The fundamental frequencies of system side and output side are $50 \mathrm{~Hz}$ and $60 \mathrm{~Hz}$, respectively.

Figure 7 shows the interharmonics analysis results of current wave of phase $B$ of the supply system side ( 5 cycles of samples). The components in the signal are measured by the TRMUSIC algorithm, which the tunable factor $\Delta$ is set to 10 . The frequencies of characteristic harmonics in system side are $(6 k \pm 1) f_{m} \quad\left(f_{m}\right)$ is the fundamental frequency of system side). It can be seen from Figure 7 that the system side of $50-60 \mathrm{~Hz} \mathrm{AC} / \mathrm{DC} / \mathrm{AC}$ converter system includes not only the characteristic harmonics of $50 \mathrm{~Hz}, 250 \mathrm{~Hz}$, and $350 \mathrm{~Hz}$, but also the interharmonics of $10 \mathrm{~Hz}, 110 \mathrm{~Hz}, 310 \mathrm{~Hz}$, and $410 \mathrm{~Hz}$, although the amplitudes of some interharmonics are small.

Then, the results of the TRMUSIC algorithm are compared with that of the MUSIC and DFT algorithm. For this simulation, we can see that the frequency analysis precision of the TRMUSIC algorithm is higher than that of the MUSIC and DFT algorithm, because the frequency resolution of the MUSIC and DFT algorithm is $10 \mathrm{~Hz}$ while that of TRMUSIC algorithm is $1 \mathrm{~Hz}$, respectively. In Figure 7, when the frequencies don't locate closest to the value of the integer frequency, the estimations with the TRMUSIC algorithm are quite accurate by predetermining the proper tunable factor $\Delta$. Unfortunately, this may result in false frequency components with the MUSIC and DFT algorithm, and it requires a longer data record. From the simulation, it is shown that the TRMUSIC algorithm indeed has a clearly higher frequency analysis precision than the MUSIC and DFT algorithm.

\subsection{Comparison with MUSIC and DFT Algorithm}

If fast Fourier transform (FFT) algorithm is used to compute its DFT, one such limitation is the power-of-two rule, requiring the number of input samples to be an integer power of two (i.e., 128, 256, 512). Therefore, choosing to lower sampling frequencies for better resolution is no longer a viable option. A clever engineer would simply increase the number of samples being taken. However, this solution quickly gets out of hand. In spite of this, the TRMUSIC algorithm may never be faster than the DFT algorithm. 


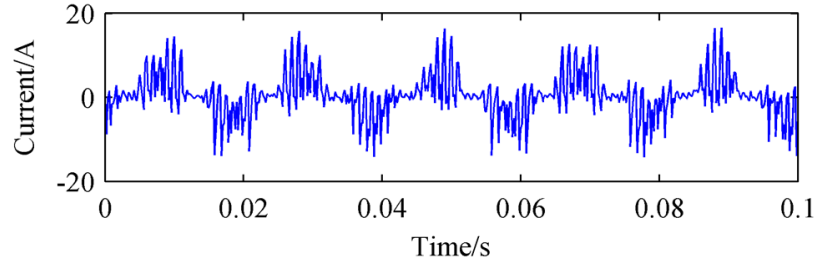

(a)

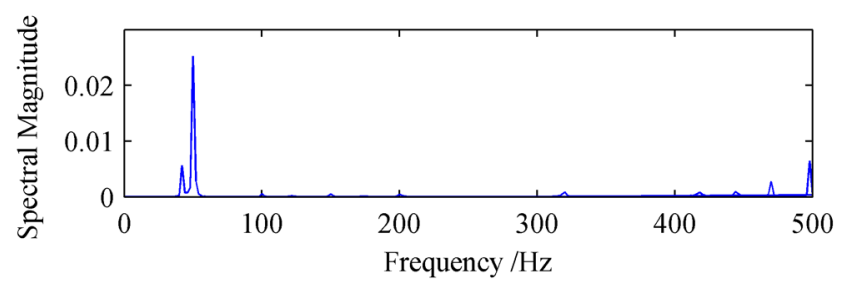

(c)

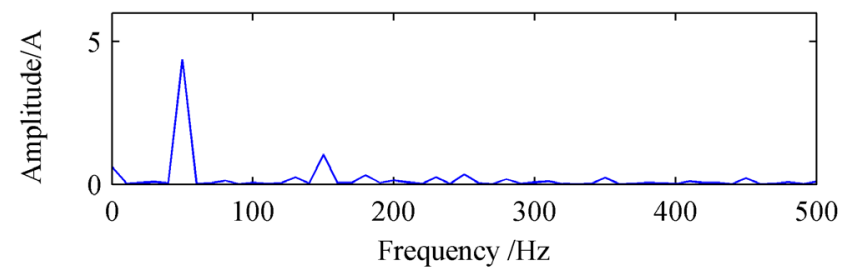

(b)

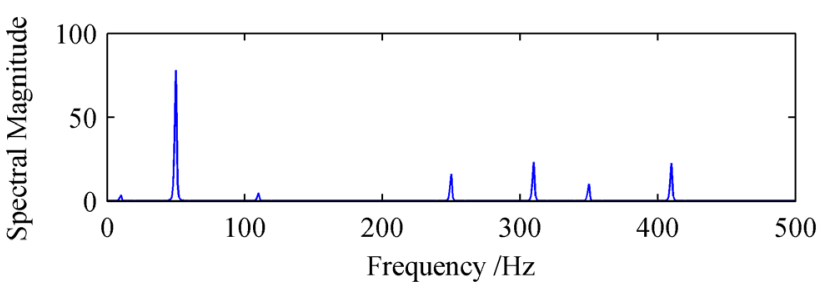

(d)

Figure 7. Interharmonics analysis of $50-60 \mathrm{~Hz}$ AC/DC/AC converter system: (a) Current wave of phase B in system side; (b) DFT spectrum; (c) MUSIC spectrum; (d) TRMUSIC spectrum.

Compared to the traditional MUSIC algorithm, the TRMUSIC algorithm is much more flexible. Given the required frequency resolution of interharmonic analysis, you can choose the proper tunable factor $\Delta$. Having expended the effort on increasing the accuracy, the TRMUSIC algorithm can be carried out effectively.

\section{Conclusions}

This paper proposes an effective method to estimate the parameters of interharmonics in power systems. With the increase of points in time domain, the frequency resolution is improved because the frequency resolution of MUSIC algorithm is $f_{s} / N$ while that of TRMUSIC algorithm is $f_{s} /(\Delta \cdot N)$. Moreover, the frequency resolution of TRMUSIC algorithm can be adapt adjusted by changing the tunable factor $\Delta$.

This research is very fundamental as an application to interharmonic analysis. Many tests were made in this work and the TRMUSIC algorithm is the most suitable to be used when estimating interharmonic spectrum. It gives us a handy solution for some drawbacks that can be found in methods like the DFT or traditional MUSIC algorithm.

The TRMUSIC algorithm really meets the need of offline applications. Furthermore, if this algorithm can be implemented in parallel computation, it should meet the need of online applications and be more practical.

\section{Acknowledgements}

This work is supported by National Natural Science Foundation of China (No. 51477124).

\section{References}

[1] IEEE (1992) IEEE Recommended Practices and Requirements for Harmonic Con- 
trol in Electric Power Systems. IEEE, New York.

[2] IEC (2002) General Guide on Harmonics and Interharmonics Measurements and Instrumentation for Power Supply Systems and Equipment Connected Thereto. IEC, Geneva.

[3] Edison, B. and Alpine, M. (2016) An Evaluation of the Extent of Correlation between Inter-Harmonic and Voltage Fluctuation Measurements. IEEE Transactions on Power Delivery, 31, 753-760. https://doi.org/10.1109/TPWRD.2015.2480715

[4] Lin, H.C. (2016) Identification of Interharmonics Using Disperse Energy Distribution Algorithm for Flicker Troubleshooting. IET Science, Measurement \& Technology, 10, 786-794. https://doi.org/10.1049/iet-smt.2016.0110

[5] Sun, Z., He, Z., Bang, T. and Li, Y. (2016) Multi-Interharmonic Spectrum Separation and Measurement under Asynchronous Sampling Condition. IEEE Transactions on Instrumentation and Measurement, 65, 1902-1912. https://doi.org/10.1109/TIM.2016.2562278

[6] Chen, C. and Chen, Y. (2014) Comparative Study of Harmonic and Interharmonic Estimation Methods for Stationary and Time-Varying Signals. IEEE Transactions on Industrial Electronics, 61, 397-404. https://doi.org/10.1109/TIE.2013.2242419

[7] Hui, J., Yang, H., Bu, W. and Li, Y. (2012) A Method to Improve the Interharmonic Grouping Scheme Adopted by IEC Standard 61000-4-7. IEEE Transactions on Power Delivery, 27, 971-979. https://doi.org/10.1109/TPWRD.2012.2183394

[8] Schmidt, R.O. (1986) Multiple Emitter Location and Signal Parameter Estimation. IEEE Transactions on Antennas and Propagation, 34, 276-280. https://doi.org/10.1109/TAP.1986.1143830

[9] Lobos, T., Napoleonic, Z., Reamer, J. and Schemer, P. (2000) High-Resolution Spectrum-Estimation Methods for Signal Analysis in Power Systems. IEEE Transactions on Instrumentation and Measurement, 55, 219-225. https://doi.org/10.1109/TIM.2005.862015

[10] Leonowicz, Z., Lobos, T. and Rezmer, J. (2003) Advanced Spectrum Estimation Methods for Signal Analysis in Power Electronics. IEEE Transactions on Industrial Electronics, 50, 514-519. https://doi.org/10.1109/TIE.2003.812361

[11] Kaveh, M. and Barabell, A.J. (1986) The Statistical Performance of the MUSIC and the Minimum-Norm Algorithms in Resolving Plane Waves in Noise. IEEE Transactions on Acoustics, Speech and Signal Processing, 34, 331-341. https://doi.org/10.1109/TASSP.1986.1164815

[12] Pisarenko, V. (1973) The Retrieval of Harmonics from a Covariance Function. Geophysical Journal of the Royal Astronomical Society, 33, 347-366. https://doi.org/10.1111/j.1365-246X.1973.tb03424.x

[13] Gu, J.F. and Wei, P. (2007) Joint SVD of Two Cross-Correlation Matrices to Achieve Automatic Pairing in 2-DAngle Estimation Problems. IEEE Antennas \& Wireless Propagation Letters, 6, 553-556. https://doi.org/10.1109/LAWP.2007.907913

[14] Hasan, M.K., Fattah, S.A. and Khan, M.R. (2003) Identification of Noisy AR Systems Using Damped Sinusoidal Model of Auto-Correlation Function. IEEE Signal Processing Letters, 10, 157-160. https://doi.org/10.1109/LSP.2003.811590

[15] Li, C., Xu, W. and Tayjasanant, T. (2003) Interharmonics: Basic Concepts and Techniques for Their Detection and Measurement. Electric Power Systems Research, 66, 39-48. https://doi.org/10.1016/S0378-7796(03)00070-1 
Submit or recommend next manuscript to SCIRP and we will provide best service for you:

Accepting pre-submission inquiries through Email, Facebook, LinkedIn, Twitter, etc. A wide selection of journals (inclusive of 9 subjects, more than 200 journals)

Providing 24-hour high-quality service

User-friendly online submission system

Fair and swift peer-review system

Efficient typesetting and proofreading procedure

Display of the result of downloads and visits, as well as the number of cited articles Maximum dissemination of your research work

Submit your manuscript at: http://papersubmission.scirp.org/

Or contact jpee@scirp.org 